\title{
SOBREPESO Y OBESIDAD PREGESTACIONAL COMO FACTOR DE RIESGO DE CESÁREA Y COMPLICACIONES PERINATALES
}

\author{
María De la Calle FM. ${ }^{1}$, Onica Armijo L. ${ }^{1}$, Elena Martín B. ${ }^{1}$, Marta Sancha N. ${ }^{1}$, \\ Fernando Magdaleno D. ${ }^{1}$, Félix Omeñaca T. ${ }^{2}$, Antonio González G. ${ }^{1}$
}

${ }^{1}$ Servicio de Obstetricia y Ginecología, ${ }^{2}$ Servicio de Pediatría, Hospital La Paz, Madrid, España.

\section{RESUMEN}

Objetivo: Comprobar si el sobrepeso y la obesidad materna son factores de riesgo de complicaciones perinatales. Método: Estudio transversal de 1.223 gestantes nulíparas atendidas en el Hospital La Paz entre agosto 2007 y abril 2008. Las gestantes se dividieron en tres grupos según el índice de masa corporal (IMC) al inicio del embarazo: 482 con peso normal (IMC: $18,5-24,9 \mathrm{Kg} / \mathrm{m}^{2}$ ); 419 con sobrepeso (IMC: $25-$ $29,9 \mathrm{Kg} / \mathrm{m}^{2}$ ) y 322 con obesidad (IMC: $\geq 30 \mathrm{Kg} / \mathrm{m}^{2}$ ). Se estudiaron las complicaciones obstétricas, semanas de gestación en el momento del parto, tipo de parto y peso fetal. Resultados: El riesgo de cesárea en las gestantes con sobrepeso fue casi el doble que el de las gestantes de peso normal (OR: 1,9; IC95\% 1,4-2,5) Las gestantes obesas sufrieron el triple de cesáreas que las de peso normal (OR: 3,1; IC95\% 2,8-4,3). El número de inducciones del parto fue mayor en las gestantes con sobrepeso (OR: 1,7; IC95\% 1,4-1,9) y el doble en las obesas (OR: 2,0; IC95\% 1,8-2,9) con respecto a las de peso normal. El riesgo de macrosomía fetal (>4.000 gramos) fue mayor en las gestantes con sobrepeso (OR: 1,5; IC95\% 1,4-2,2) y en las obesas (OR: 1,9; IC95\% 1,3-2,8) que en las de peso normal. En el análisis de regresión logística multivariante (ajustando por edad, diabetes e hipertensión gestacionales) persistió la asociación entre sobrepeso y cesárea (OR: 2,1; IC95\% 1,7-2,7) y entre obesidad y cesárea (OR: 3,0; IC95\% 2,6-3,8). Conclusiones: El sobrepeso y la obesidad materna al inicio del embarazo están asociados con un aumento del número de cesáreas y de inducciones del parto. Este riesgo es mayor a medida que aumenta el IMC materno.

\section{PALABRAS CLAVE: Sobrepeso, obesidad, gestación, cesárea, inducción del parto}

\section{SUMMARY}

Objective: The objective of this study is to determine if the maternal prepregnancy overweight and obesity are related to risk of cesarean delivery and other obstetrical complications. Methods: Transversal study of 1223 nulliparous women that delivered in our maternity between August 2007 and April 2008. Women were categorized by the body mass index (BMI): 482 normal weight (BMI: $18.5-24.9 \mathrm{Kg} / \mathrm{m}^{2}$ ), 419 overweight (BMI: $25-29.9 \mathrm{Kg} / \mathrm{m}^{2}$ ) and 322 obesity (BMl: $\left.\geq 30 \mathrm{Kg} / \mathrm{m}^{2}\right)$. Obstetrical complications, labor and its complications, and neonatal weight were studied. Results: Women who were overweight was nearly twice as likely to deliver by cesarean section as women with normal weight (OR: 1.8; $95 \% \mathrm{Cl} 1.4-2.9$ ). Obese women experienced a three-fold increase in risk of cesarean section in comparison to normal weight women (OR: 3.02; $95 \% \mathrm{Cl}$ 2.6-4.5). Number of labor inductions was higher in overweight and obese group (OR: $1.6 ; 95 \% \mathrm{Cl}$ 1.3-1.9) and (OR: $2.1 ; 95 \% \mathrm{Cl} 1.7-2.9)$ respectively in comparison to control group. The highest risk of fetal macrosomia (weight $>4000 \mathrm{~g}$ ) was in the overweight (OR: 1.5; 95\% Cl 1.4-2.2) and obese women (OR: 1.9; 
$95 \% \mathrm{Cl}$ 1.3-2.8) compared with the normal weight women. In multivariate logistic regression analysis, the association persisted between obesity and cesarean delivery (OR 3.0; 95\% Cl 2.6-3.8) and among overweight and cesarean section (OR $2.1 ; 95 \% \mathrm{Cl} 1.7-2.7)$ after adjusting for variables such as age, diabetes and hypertension. Conclusions: Overweight or obesity prior to pregnancy are associated with an increased risk of cesarean delivery and labor inductions. This risk increases as the BMI increases.

KEY WORDS: Obesity, overweight status, pregnancy, cesarean delivery, induction of labour

\section{INTRODUCCIÓN}

En las mujeres gestantes el sobrepeso y la obesidad se han asociado a un mayor número de complicaciones durante el embarazo, como son la diabetes gestacional, la enfermedad hipertensiva del embarazo, la preclampsia, las infecciones maternas (urinarias ó endometritis), la enfermedad tromboembólica, el asma y la apnea del sueño. La macrosomía y el trauma fetal durante el parto son también más frecuentes en los hijos de madres con sobrepeso y obesidad que en los de madres de peso normal (1-5).

Durante el proceso del parto, también aumentan las complicaciones obstétricas en las gestantes con mayor índice de masa corporal (IMC). Varios estudios demuestran un aumento en el número de embarazos cronológicamente prolongados, inducciones del parto, horas de dilatación, partos instrumentales y el número de cesáreas (2-9). La mayoría de las cesáreas realizadas en las gestantes obesas son por no progresión de parto o desproporción céfalo-pélvica (5-9). Además se ha descrito que el riesgo de cesárea aumenta conforme lo hace el IMC $(2,3,5,9)$.

En el presente estudio, se ha querido comprobar si el sobrepeso y la obesidad materna pregestacional están asociados a un incremento del riesgo de complicaciones durante el parto y al aumento del número de cesáreas en una población española de gestantes nulíparas.

\section{MATERIAL Y MÉTODO}

Estudio transversal de 1.223 gestantes nulíparas que se atendieron en el Hospital la Paz entre los meses de agosto de 2007 y abril de 2008. Se determinó el IMC de cada paciente calculando el peso en kilogramos al inicio del embarazo y la altura en centímetros, utilizando la fórmula Peso/ Altura2 $\left(\mathrm{Kg} / \mathrm{m}^{2}\right)$. Se dividió a las gestantes en tres grupos según los criterios establecidos por The National Institutes of Health and the World Health Organization: peso normal (IMC: $18,5-24,9 \mathrm{Kg} / \mathrm{m}^{2}$ ), sobrepeso (IMC: $25-29,9 \mathrm{Kg} / \mathrm{m}^{2}$ ) y obesidad (IMC: $\left.\geq 30 \mathrm{Kg} / \mathrm{m}^{2}\right)$ (10). El número de gestantes con peso normal fueron 482, con sobrepeso 419 y obesidad 322. Se consideró como grupo control las gestantes con peso normal.

Las variables estudiadas fueron: edad materna, hábito tabáquico (fumadoras activas), diabetes gestacional, hipertensión arterial gestacional, preclampsia, kilos de peso incrementados durante el embarazo, semanas de gestación en el momento del parto, inducción del parto, horas de dilatación y vía del parto: eutócico, parto instrumental (forceps o espátulas) y cesárea. Se estudiaron los distintos motivos de la cesárea: riesgo de pérdida de bienestar fetal, no progresión de parto o desproporción céfalo-pélvica.

Con relación al peso neonatal, se consideró macrosomía fetal cuando el peso era mayor a 4.000 gramos. Embarazo cronológicamente prolongado al tiempo de gestación $\geq 41$ semanas +3 días. Diabetes gestacional cuando la sobrecarga oral de glucosa de 50 gramos era a las 2 horas $\geq 140 \mathrm{mg} / \mathrm{dl} \mathrm{y}$ la sobrecarga oral de glucosa de 100 gramos tenía dos resultados patológicos. Hipertensión arterial gestacional cuando la tensión arterial diastólica era $\geq 90 \mathrm{mmHg}$ y/o la tensión arterial sistólica era $\geq 140$ $\mathrm{mmHg}$ después de la semana 20 de gestación y la proteinuria en orina de 24 horas inferior a 300 $\mathrm{mg} / \mathrm{L}$. Se consideró preeclampsia cuando cuando la tensión arterial diastólica era $\geq 90 \mathrm{mmHg}$ y/o la tensión arterial sistólica era $\geq 140 \mathrm{mmHg}$ después de la semana 20 de gestación y proteinuria mayor a $300 \mathrm{mg} / \mathrm{L}$ en 24 horas.

Se excluyeron las gestantes con un IMC $<18,5$ $\mathrm{Kg} / \mathrm{m}^{2}$, las multíparas, gestaciones múltiples, gestaciones sin control, gestaciones controladas a partir de la semana 16, diabetes pregestacional, enfermedades maternas (cardiacas, renales, trombofilias, infección por VIH), malformaciones fetales, presentaciones anómalas y gestantes con cirugías uterinas previas.

Análisis estadístico: Los datos se analizaron en el Servicio de Estadística del Hospital La Paz mediante el programa SPSS 9. La descripción de los 
datos cualitativos se realizó en forma de frecuencias absolutas y porcentajes, y los datos cuantitativos mediante la media y la desviación estándar ( $\pm \mathrm{DE})$.

La diferencia entre casos y controles se analizaron mediante los test t-Student y $x^{2}$, según el tipo de variable. Las comparaciones múltiples se hicieron mediante el método de Dunn-Sidák. Se realizó un análisis multivariante consistente en una regresión logística por pasos hacia delante para calcular la relación entre obesidad y cesárea, y entre sobrepeso y cesárea, ajustando por edad, y por hipertensión arterial y diabetes gestacionales. Una regresión logística similar a la previa se realizó para evaluar la relación entre obesidad e inducción del parto, y sobrepeso e inducción del parto, ajustando por las mismas variables. La magnitud de la asociación se expresa en forma de odds ratio (OR) con intervalo de confianza (IC) del 95\%. Todas las pruebas estadísticas se han considerado bilaterales y como valores significativos los que presentaban $p<0,05$.

Todas las pacientes fueron informadas del estudio y de su anonimato si decidían acceder a participar. El $100 \%$ de las gestantes accedieron a participar en el estudio y firmaron un consentimiento informado.

\section{RESULTADOS}

Con respecto a las características demográficas, no se encontraron diferencias significativas en relación a la edad entre los tres grupos, siendo el rango entre los 17 y los 41 años. Tampoco había diferencias en el hábito tabáquico. En cuanto al peso fetal, fue superior en las gestantes con sobrepeso y obesidad que en las de peso normal. La macrosomía fetal, definido como un peso mayor a 4.000 gramos, fue más frecuente en las gestantes con sobrepeso (OR: 1,5; IC 95\% 1,4-2,2) y en las ges- tantes obesas (OR: 1,9; IC 95\% 1,3-2,8) en comparación con las gestantes de peso normal (Tabla I).

En relación a las complicaciones maternas, la diabetes gestacional fue más frecuente en las gestantes con sobrepeso y obesidad pregestacional que en las de peso normal. El riesgo de diabetes gestacional fue mayor en las mujeres con sobrepeso (OR: 1,6; IC 95\% 1,4-2,0) y aún más elevado en mujeres obesas (OR: 2,2; IC 95\% 1,9-3) en relación con las gestantes de peso normal. El riesgo de desarrollar hipertensión arterial en las gestantes con sobrepeso (OR: 1,8; IC 95\% 1,6-2,1) y obesidad (OR: 2,3; IC 95\% 1,8-2,7) fue mayor que en las de peso normal. El riesgo de preclampsia fue también mayor en las gestantes con sobrepeso (OR: 1,2; IC $95 \% 1,1-1,7$ ) y obesidad (OR: 1,8; IC 95\% 1,5-2,2) con respecto al grupo control. Los kilogramos de peso incrementados durante el embarazo no tenían relación con el IMC al inicio del mismo, aunque se observaba una tendencia a la menor ganancia de peso en las mujeres obesas en relación a las de peso normal (Tabla II).

En cuanto a las complicaciones en el momento del parto, las semanas de gestación y el porcentaje de embarazos cronológicamente prolongados $(\geq 41+3$ semanas) no fueron diferentes entre los tres grupos. Si embargo, se observó una tendencia hacia un mayor número de semanas y de embarazos cronológicamente prolongados en el grupo de gestantes con sobrepeso y obesidad. El número de inducciones del parto fue mayor en las gestantes con sobrepeso (OR: 1,7; IC 95\%, 1,4-1,9) en relación al grupo control y fue el doble en gestantes obesas en comparación con las de peso normal (OR: 2,0; IC 95\% 1,8-2,9). Las horas de dilatación fueron superiores en las gestantes con sobrepeso y en las obesas con respecto al grupo control (Tabla III).

\section{Tabla I}

CARACTERÍSTICAS DEMOGRÁFICAS DE LAS GESTANTES SEGÚN CONDICIÓN NUTRICIONAL

\begin{tabular}{|c|c|c|c|c|}
\hline & $\begin{array}{l}\text { N ormal } \\
\mathrm{n}: 482\end{array}$ & $\begin{array}{l}\text { Sobrepeso } \\
\mathrm{n}: 419\end{array}$ & $\begin{array}{l}\text { Obesidad } \\
\text { n: } 322\end{array}$ & Valor $p$ \\
\hline Edad (años) & $28,5 \pm 5,1$ & $29,6 \pm 5,3$ & $27,5 \pm 5,2$ & NS \\
\hline Tabaco (\%) & 31,8 & 34,1 & 30,6 & NS \\
\hline Peso fetal (g) & $3.394 \pm 501^{\mathrm{ab}}$ & $3.478 \pm 562$ & $3.990 \pm 657$ & 0,01 \\
\hline Macrosomía (\%) & $6,8^{\mathrm{ab}}$ & $9,0^{\mathrm{b}}$ & 14,4 & 0,001 \\
\hline
\end{tabular}

a: $p<0,05$ en comparación con sobrepeso. b: $p \leq 0,01$ en comparación con obesidad. NS: no significativo. 
Tabla II

COMPLICACIONES MATERNAS DURANTE EL EMBARAZO SEGÚN CONDICIÓN NUTRICIONAL

\begin{tabular}{|c|c|c|c|c|}
\hline & $\begin{array}{l}\text { Normal } \\
\mathrm{n}: 482\end{array}$ & $\begin{array}{l}\text { Sobrepeso } \\
\text { n: } 419\end{array}$ & $\begin{array}{l}\text { Obesidad } \\
\text { n:322 }\end{array}$ & Valor $p$ \\
\hline Diabetes gestacional (\%) & $2,4^{\mathrm{ab}}$ & $5,7^{\mathrm{b}}$ & 9,3 & 0,001 \\
\hline Hipertensión arterial (\%) & $3,6^{\mathrm{ab}}$ & 9,5 & 11,1 & 0,001 \\
\hline Preeclampsia (\%) & $1,8^{\mathrm{ab}}$ & 3,2 & 5,7 & 0,01 \\
\hline $\begin{array}{l}\text { Incremento de peso en el } \\
\text { embarazo }(\mathrm{Kg})\end{array}$ & $12 \pm 7$ & $10 \pm 5$ & $6 \pm 5$ & NS \\
\hline
\end{tabular}

$a: p<0,01$ en comparación con sobrepeso. b: $p=0,01$ en comparación con obesidad. NS: no significativo.

Tabla III

CARACTERÍSTICAS DEL PARTO DE LAS GESTANTES SEGÚN CONDICIÓN NUTRICIONAL

\begin{tabular}{lllll}
\hline & $\begin{array}{l}\text { Normal } \\
\mathrm{n}: 482\end{array}$ & $\begin{array}{l}\text { Sobrepeso } \\
\mathrm{n}: 419\end{array}$ & $\begin{array}{l}\text { Obesidad } \\
\mathrm{n}: 322\end{array}$ & Valor $\mathrm{p}$ \\
\hline $\begin{array}{l}\text { Edad gestacional al parto } \\
\text { (semanas) }\end{array}$ & $38,3 \pm 3,2$ & $39,2 \pm 2,1$ & $39,5 \pm 3,4$ & $\mathrm{NS}$ \\
$\begin{array}{l}\text { Embarazo prolongado (\%) } \\
\text { Inducción del parto (\%) }\end{array}$ & 14,6 & 17,4 & 19,3 & $\mathrm{NS}$ \\
Horas de dilatación & $19^{\mathrm{a}, \mathrm{b}}$ & $26^{\mathrm{c}}$ & 47 & 0,001 \\
Parto instrumental (\%) & $4 \pm 2^{\mathrm{a}, \mathrm{c}}$ & $7 \pm 3$ & $8 \pm 2$ & 0,01 \\
Cesárea (\%) & $25,2^{\mathrm{a}, \mathrm{c}}$ & 28,5 & 29,1 & 0,02 \\
\hline
\end{tabular}

$a: p<0,05$ en comparación con sobrepeso. b: $p=0,001$ en comparación con obesidad. $c$ : $p<0,05$ en comparación con obesidad. NS: no significativo.

Los partos instrumentales fueron mayores en las gestantes con sobrepeso (OR: 1,5; IC 95\% 1,3$1,9)$ y obesidad (OR: 1,8 ; IC $95 \% 1,5-2,2)$ respecto a las mujeres con peso normal (Tabla III).

Las gestantes obesas y con sobrepeso tuvieron una incidencia de cesáreas mayor que las gestantes con peso normal (Tabla III). El riesgo de cesárea en las gestantes con sobrepeso fue practicamente el doble con respecto a las de peso normal (OR: 1,9 ; IC 95\% 1,4-2,5). Las mujeres obesas sufrieron el triple de cesáreas que las de peso normal (OR: 3,1; IC 95\% 2,8-4,3), como puede observarse en la Tabla IV. Los motivos de la cesáreas fueron: $72 \%$ por no progresión de parto, $21 \%$ por desproporción pélvico cefálica y $7 \%$ por riesgo de pérdida de bienestar fetal.
Tabla IV

INCIDENCIA DE INDUCCIÓN DEL PARTO Y DE CESÁREA SEGÚN CONDICIÓN NUTRICIONAL

\begin{tabular}{lll}
\hline $\begin{array}{l}\text { Condición } \\
\text { nutricional }\end{array}$ & $\begin{array}{l}\text { Inducción del parto } \\
\text { OR (IC95\%) }\end{array}$ & $\begin{array}{l}\text { Cesárea } \\
\text { OR (IC95\%) }\end{array}$ \\
\hline $\begin{array}{l}\text { Sobrepeso } \\
\text { n: } 419\end{array}$ & $1,8(1,4-2,3)$ & $2,1(1,7-2,7)$ \\
$\begin{array}{l}\text { Obesidad } \\
\text { n: } 322\end{array}$ & $2,2(1,8-2,7)$ & $3,0(2,6-3,8)$ \\
\hline
\end{tabular}

Análisis de regresión logística multivariante, ajustando por edad y diabetes gestacional e hipertensión gestacional. OR: odds ratio en comparación con gestantes de peso normal. IC: intervalo de confianza 
Cuando se realizó un análisis multivariante, ajustando por posibles variables de confusión, como son la edad y la hipertensión y diabetes gestacionales, se comprobó que el riesgo de cesárea seguía asociado al sobrepeso (OR: 2,1; IC 95\% $1,7-2,7$ ) y a la obesidad (OR: 3,0 ; IC $95 \% 2,6-3,8$ ). Así mismo, se asociaba el riesgo de inducción del parto con el sobrepeso (OR: 1,8; IC 95\% 1,4-2,3) y la obesidad (OR: 2,2; IC 95\% 1,8-2,7).

\section{DISCUSIÓN}

El impacto de la obesidad sobre el momento del parto ha sido estudiado en distintas poblaciones del mundo. Sin embargo, este es el primer estudio que se realiza en España, donde la población obesa en edad reproductiva ha ido aumentando a lo largo de los años.

Hemos encontrado una relación lineal entre el aumento del IMC al inicio del embarazo y el riesgo de desarrollar diabetes gestacional, hipertensión gestacional, preclampsia, inducciones del parto y cesáreas. Estos riesgos son independientes del peso ganado durante el embarazo. Estudios previos han puesto también de manifiesto la relación entre la obesidad materna y las complicaciones durante el embarazo como la diabetes gestacional, la hipertensión y la preclampsia (1-8). Nuestros resultados son similares a los encontrados por otros autores $(1,2)$, aunque en otros estudios el riesgo de estas complicaciones ha sido aún mayor $(3,8)$. Una de las teorías del aumento de diabetes gestacional, hipertensión y preeclampsia en las gestantes obesas es el aumento de la leptina, proteína circulante producida por el adipocito y también por la placenta durante el embarazo (9). El aumento de la producción de leptina placentaria en las gestantes obesas produce alteraciones de metabolismo de los hidratos de carbono y daño endotelial que están en relación con la diabetes y la preclampsia respectivamente $(11,12)$.

Con respecto a las complicaciones durante el parto, nuestro estudio coincide con otros previos en los que se demuestra un incremento de las inducciones del parto en las gestantes con sobrepeso y obesidad pregestacional (1-5,8). El riesgo de inducción del parto fue aumentando conforme lo hacía el IMC. Algunos autores demuestran un aumento del embarazo cronológicamente prolongado, subsidiario de inducción, en las gestantes con sobrepeso y obesidad (4). En nuestro estudio, al igual que otros autores $(3,8)$, no encontramos una prolongación en las semanas de gestación en el momento del parto ni un aumento de embarazos cronológicamente prolongados. Los factores que controlan el retraso en el inicio de las contracciones del parto en las pacientes obesas, no son aún bien conocidos. Unos autores describen una dificultad de contracción del útero en las gestantes obesas (12) y otros demuestran una disminución en los niveles plasmáticos de cortisol en estas gestantes respecto a las de peso normal, lo que reduce la producción de hormona liberadora de cortocotropina placentaria, prolongando así el inicio de las contracciones (13). Además, las mujeres obesas presentan en el tejido adiposo un aumento de estrógenos que produce una disminución de sus niveles plasmáticos y altera la relación estrógeno/progesterona en el plasma materno, enlenteciendo la aparición de la dinámica uterina (14).

En relación al tipo de parto, en el presente estudio se demuestra un aumento del riesgo de partos instrumentales y de cesáreas en las gestantes con sobrepeso y obesidad con respecto a las de peso normal, que se incrementa conforme lo hace el IMC, como también han puesto de manifiesto en estudios realizados en otros países (1-5,8,15-17). En el presente estudio, al igual que en otro previo (2), se encontró el doble de riesgo de cesárea en las gestantes con sobrepeso y el triple de riesgo en las obesas en relación a las de peso normal. Sin embargo, otros autores describen hasta seis veces más riesgo de cesárea en las gestantes obesas (16) $y$ otros no encuentran un riesgo tan elevado como en nuestro estudio $(1,8,17)$. Una de las posibles razones para explicar la heterogenicidad de los resultados es la diferente definición de obesidad, $\geq 29$ $\mathrm{Kg} / \mathrm{m}^{2}$ en unos estudios $\mathrm{y} \geq 30 \mathrm{Kg} / \mathrm{m}^{2}$ en otros, así como la diferente definición de peso normal que en algunas publicaciones se ha considerado hasta de $26 \mathrm{Kg} / \mathrm{m}^{2}(1-4,8)$.

La causa más frecuente de cesárea en nuestro estudio es la no progresión de parto, seguida de la desproporción céfalo-pélvica, resultados que concuerdan con otros autores (8). Esto puede explicarse por una disminución de las contracciones uterinas durante el parto, debido a que en las gestantes obesas se ha descrito tanto un descenso en la producción de la hormona liberadora de corticotropina como un descenso en los niveles de estrógenos $(13,14)$. En el presente estudio, las gestantes con sobrepeso y las obesas tuvieron más horas de dilatación que las de peso normal. También en otros estudios se ha comprobado que las mujeres con sobrepeso y obesidad están más horas de parto $(18,19)$. Algunos autores especulan, que un factor contribuyente a la prolongación del parto en las mujeres obesas es un exceso de tejido blando en la pelvis materna, que dificultaría el encajamiento fetal (17). 
Con respecto a los resultados fetales, se demostró un aumento del peso fetal y de macrosomía fetal en las gestantes con sobrepeso y obesas con respecto a las de peso normal. El riesgo de macrosomía fetal va aumentando conforme lo hace el IMC. Estos mismos resultados han sido corroborados por otros autores y son independientes de los kilos de peso ganados en el embarazo y de la diabetes gestacional $(1,3,8)$. Es importante a la hora del parto que el obstetra conozca el mayor riesgo de macrosomía fetal que tienen las pacientes obesas, para poder prevenir y actuar ante complicaciones derivadas de la misma.

Los resultados de este estudio, con un aumento de complicaciones obstétricas, de inducciones del parto y del número de cesáreas en relación con el aumento del IMC, podría paliarse con información preconcepcional, insistiendo en el cambio de hábitos nutricionales y de estilo de vida a las gestantes con sobrepeso y obesidad que estén planeando un embarazo.

\section{BIBLIOGRAFÍA}

1. Weiss JL, Malone FD, Emig D, Ball RH, Nyberg DA, Comstock $\mathrm{CH}$, et al. Obesity, obstetrics complications and cesarean delivery rate. A population-based screening study. Am J Obstet Gynecol 2004;190:1091-7.

2. Dempsey JC, Ashiny Z, Qu CF, Miller RS, Sorensen TK, Williams MA. Maternal pre-pregnancy overweight status and obesity as risk factors for cesarean delivery. J Mater Fetal Neonat Med 2005;17:179-85.

3. Bhattacharya S, Campbell DM, Liston WA, Bhattacharya. Effect of body mass index on pregnancy outcomes in nulliparous women delivering singleton babies. BMCPublic Health 2007;7:1-8.

4. Denison FC, Price J, Graham C, Wild S, Liston WA. Maternal obesity, length of gestation, risk of postdates pregnancy and spontaneous onset of labour at term. BJOG 2008;720-5.

5. Abenheim HA, Kinch RA, Morin L, Benjamin A, Usher $R$. Effect of prepregnancy body mass index categories on obstetrical and neonatal outcomes. Arch Gynecol Obstet 2007;275:39-43.

6. Krishnamoorthy $\mathrm{U}$, Schram $\mathrm{CMH}$, Hill SR. Maternal obesity in pregnancy: is term for meaningful resear- ch to inform preventive and management strategies? BJOG 2006;113:1134-40.

7. O'Brien TE, Ray JG, Chan WS. Maternal body mass index and the risk of preclampsia: a systematic review. Epidemiology 2003;14:368-74.

8. Ducarme G, Rodrigues A, Aissaoui F, Davitian C, Pharisien I, Uzan M. Grossesse des patientes obéses: quels risques faut-il craindre? Gynecologie Obstetrique Fertilite 2007;35:19-24.

9. Lepercq J, Catalano P, Hauguel de Monzon S. Leptine et grossesse: dogmes, questions et perspectives. Gynecolgie Obstetrique Fertilite 2007;35:89-95.

10. Manderson J, Patterson C, Hadden D, Traub A, Leslie $\mathrm{H}$, McCance $\mathrm{D}$. Leptin concentrations in maternal serum and cord blood in diabetic and nondiabetic pregnancy. Am J Obstet Gynecol 2003;188:1326-32.

11. Mise $H$, Sagawa $N$, Matsumoto T, Yura $S$, Nanno $H$, Itoh $\mathrm{H}$, et al. Augmented placental production of leptin in preclampsia: possible involvement of placental hypoxia. J Clin Endocrinol Metab 1998;83:3225-9.

12. Zhang J, Bricker L, Wray S, Quenby S. Poor uterine contractility in obese women. BJOG 2007;114:343-8.

13. Emanuel RL, Robinson BG, Seely EW, Graves SW, Kohane I, Saltzman D, et al. Corticotrophin releasing hormone levels in human plasma and amniotic fluid during gestation. Clin Endocrinol (Oxf) 1994;40:25762.

14. Smith R, Mesiano S, McGrath S. Hormone trajectories leading to human birth. Regul Pept 2002;108:159-64.

15. Rode L, Nilas L, Wojdemann K, Tabor A. Obesityrelated complications in Danish single cephalic term pregnancies. Obstet Gynecol 2005;105:537-42.

16. Young TK, Woodmansee B. Factors that are associated with cesarean delivery in a large private practice: the importante of prepregnancy body mass index and weight gain. Am J Obstet Gynecol 2002;187:312-20.

17. Crane SS, Wojtowycz MA, Dye TD, Aubry RH, Artal R. Association between pre-pregnancy obesity and the risk of cesarean delivery. Obstet Gynecol 1997;89:213-6.

18. Hamon C, Fanello S, Catala L, Parot E. Consequence de l'óbesité maternelle sur le déroulement du travail et láccouchement à léxclusion des autres pathologies pouvant modifier la prise en charge obstétricale. J Gynecol Obstet Biol Reprod (Paris) 2005;34:109-14.

19. Johnson SR, Kolberg BH, Varner MW. Maternal obesity and pregnancy. Surg Gynecol Obstet 1987;164:43137. 